\title{
Satisfaction of Nigerian patients with health services: a protocol for a systematic review
}

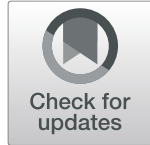

Maureen O. Akunne', Mathew J. Okonta ${ }^{1}$, Chinwe V. Ukwe', Thomas L. Heise ${ }^{2,3^{*}}$ and Obinna I. Ekwunife ${ }^{4}$

\begin{abstract}
Background: Patient-based assessment of health services is becoming popular in measuring the standard of care. Both quantitative and qualitative methods are available. Patient satisfaction surveys are commonly used to record the experiences of patients in hospitals, whereas qualitative designs (e.g., interviews and focus group discussions) are used less frequently. To date, there has been no systematic review published devoted to patient satisfaction with health services in Nigeria. We aim to (1) systematically analyze relevant quantitative studies to pinpoint excellent procedures in measuring patient satisfaction with health services, (2) to investigate if a reference method (gold standard method) exists, and (3) to identify relevant topics which are recognized by patients as important for the delivery of a high-quality health service in Nigeria.

Methods: Searches of eight electronic journal databases, including MEDLINE, EMBASE, CINAHL, PsycINFO, AJOL, CDSR, DARE, and HTA will be conducted to identify studies assessing patient satisfaction with health services in Nigeria. The searches will be supported by manual searches in reference lists of relevant primary studies and systematic reviews. The review will be limited to studies published since 2007. After a stepwise screening process by two reviewers, data from included studies will be extracted and reviewed. The COSMIN RoB checklist will be used to critically appraise included studies. We will carry out an extensive data synthesis to answer the review questions.

Discussion: The intended systematic review will provide information on how the satisfaction of patients with health services has earlier been described and assessed in Nigerian studies. It will establish if a gold standard method exists and synthesize information on topics which might be of special interest to patients. Review findings will enrich the debate on patient-centered care and overall performance of health quality standards in Nigeria.
\end{abstract}

Systematic review registration: PROSPERO CRD42018108140

Keywords: Patient satisfaction, Health services, Nigeria, Systematic review

\section{Background}

\section{The Nigerian patients and health services}

Low- and middle-income countries (LMICs) such as Nigeria commonly face the dual health burden caused by infectious (communicable) diseases (e.g., malaria, HIV/AIDS, hepatitis, tuberculosis, influenza) and non-infectious (non-communicable) diseases (e.g., diabetes mellitus, cancer, cardiovascular diseases, chronic respiratory diseases) [1]. The nation's healthcare system has also notable challenges which include

\footnotetext{
* Correspondence: heise@leibniz-bips.de

${ }^{2}$ Research Group for Evidence-Based Public Health, Institute for Public Health and Nursing Research, Health Sciences, University of Bremen, Bremen, Germany

${ }^{3}$ Leibniz Institute for Prevention Research and Epidemiology - BIPS, Bremen, Germany

Full list of author information is available at the end of the article
}

underinvestment in health systems, poor healthcare infrastructure, inadequate funding and policy frameworks, and insufficient implementations of Public-Private-Partnerships (PPPs). These, among other challenges, may contribute to poor satisfaction with health services experienced by Nigerian patients $[2,3]$.

Many Nigerian patients, those who can afford it, now receive medical treatment abroad (medical tourism), especially for general surgeries as well as disease management in the fields of cardiology, neurology, and overall cancer management. In 2012, Nigerians spent an estimated 260 million USD in India for medical treatment and related costs alone. A large proportion of travels to India (about 40\%) can be explained by medical reasons [4]. Another report also shows that Nigerians spend 1

(c) The Author(s). 2019 Open Access This article is distributed under the terms of the Creative Commons Attribution 4.0 International License (http://creativecommons.org/licenses/by/4.0/), which permits unrestricted use, distribution, and 
billion USD every year to obtain emergency medical treatments abroad [5]. This includes medical tourism to countries and geographic regions such as South Africa, Zimbabwe, and countries in the Middle East (e.g., Egypt, Israel, Turkey) which are supposed to offer better health care service compared to Nigeria. It is not as if the country does not produce the required clinical expertise, but lack of favorable circumstances and enabling environments in the nation form the basis for the persistent brain drain, not to mention abnormally low remunerations. The National Health Service (NHS) in the UK, for example, has over and above 4000 physicians employed from Nigeria, this number is even higher for Nigerian doctors working in the USA. In total, more than 25,000 physicians from Nigeria are working abroad, affecting healthcare delivery back home [6]. This, without doubt, negatively influences the standard of care and could invariably affect the satisfaction of patients in Nigeria, i.e., those who cannot afford overseas treatment.

Financing of healthcare is very important to achieving universal healthcare coverage. In Nigeria, healthcare financing occurs through a variety of sources such as revenue from taxes, direct payment for treatment (out-of-pocket payments (OOPs)), funding by donors, and health insurances (social and community). WHO, UNICEF, and USAID are some of the donor groups that have funded Nigeria healthcare projects. With the inauguration of the National Health Insurance Scheme (NHIS) in Nigeria in 2005, accessibility to healthcare was provided to many Nigerians. But since then, only those working in public (formal) sectors (less than $5 \%$ of the Nigerian workforce) have registered. Nigerian government intends to use Community-Based Health Insurance (CBHIS) for workers in the informal sector and rural areas. States such as Lagos, Anambra, Ogun, and Kwara are currently implementing CBHIS. For instance, a law was passed in Lagos establishing a Lagos State Health Insurance Scheme [4].

The slow pace of Nigeria's implementation efforts to achieve universal health insurance coverage is unfortunate because countries such as Ghana, Rwanda, and Kenyawhich started several years after Nigeria-have hit over 50\% coverage in the meantime [7]. Direct payments in the form of OOPs are the main source, comprising about $70.3 \%$ of total health expenditures in 2009 [8]. This percentage share is seen as one of the highest in the world [8].

\section{Health outcome surveys}

Definition of healthcare outcomes has been given as "measures of the result of what happens to patients as a consequence of their encounters with the healthcare system" [9]. In clinical research, three types of outcomes are measured: (a) clinical outcomes which are consequences of a medical treatment (e.g., laboratory values); (b) humanistic outcomes which are end results of a disease or a treatment on the patient's ability to perform normal daily activities and quality of life, including patient-reported outcomes (PROs) (e.g., satisfaction of patients with healthcare services); and (c) economic outcomes which are direct, indirect, and intangible costs of treatment in relation with the results of therapy options.

Traditional medical models of care had concentrated on clinical outcomes only, whereas more recent medical models of care integrate clinical outcomes as well as economic and humanistic outcomes. This new approach, the Economic, Clinical and Humanistic Outcomes is termed the ECHO model of care. The ECHO model builds on the traditional model but broadens its scope by incorporating the humanistic and economic outcomes and thus provides a more comprehensive measurement of health care value [10].

Patient satisfaction, a humanistic outcome, has been studied as a crucial element when assessing health outcomes and standard of care [11]. Often, quantitative surveys such as questionnaires have been used to document patient interactions with the healthcare system, such as their satisfaction with health services in hospitals and clinics. Qualitative designs such as interviews and focus group discussions are less often used, but can gain a more comprehensive and thorough view of patients' experiences. A study conducted in Nigeria [11] recorded high level of satisfaction of HIV patients with pharmaceutical services using a Patient Satisfaction with Pharmaceutical Service (PSPS) questionnaire [12]. However, in the study, the patients indicated low satisfaction with the item that examined the supply of penned information by pharmacists. Using a standardized structured interviewed questionnaire, Karunamoorthi et al. [13] also demonstrated that most of the respondents were satisfied with the pharmaceutical service. $82.5 \%$ of the participants in the study, however, indicated long waiting hours to finally receive their medication as a major problem that is responsible for their dissatisfaction. The research on the satisfaction of patients with health services is not only important in chronic diseases such as HIV/AIDS, but, in fact, in all areas of health service delivery. For instance, a study done in the south-west region of Nigeria on the satisfaction with primary health services also indicated waiting time and availability of drugs as the main interests of the participants [14]. It is worthwhile to note that respondents under NHIS as well as non-NHIS respondents were highly satisfied with the health services they had received from their healthcare providers at their healthcare facilities [15-19]. Contrary to the studies discussed above, Abebe et al. recorded low levels of satisfaction by the respondents with pharmaceutical services [20]. Thus, evaluation of patients' satisfaction with health services is important and crucial to pinpoint certain areas of service and practice which need to be improved. A systematic analysis of these relevant studies can also help to identify 
relevant approaches in evaluating patient satisfaction and to establish different appraisal instruments based on the patients' most important considerations.

A review focusing on the measurement of patient satisfaction in clinics specialized in the field of sexually transmitted diseases (STDs) has already been published [21]. Some of such reviews included studies which had been carried out in LMICs such as Nigeria [22, 23]. Another review examined the satisfaction measurement instruments for healthcare service users in Brazil [24]. In the latter review, the researchers concluded, based on their findings, that there was no gold standard method for assessing patient satisfaction. A review of patients' views on the quality of primary health care in sub-Saharan Africa was published in 2015. This review included six studies from Nigeria, but did not make country-specific conclusions [25]. Even though studies from Nigeria were included in systematic reviews before, none has been conducted focusing on Nigerian patients and their satisfaction with local health services. The study therefore intends to address the following questions:

1. How has patient satisfaction with health services in Nigerian hospitals been previously described and assessed?

2. Is there any existence of a gold standard method?

3. Is it possible to identify issues/topics considered by patients as most important in delivering a highquality service?

4. Are there any areas of dissatisfaction and if so, what are they?

\section{Methods}

\section{Protocol}

This protocol follows the guidelines on reporting as outlined in the Preferred Reporting Items in Systematic Reviews and Meta-analyses (PRISMA) statement [26]. The protocol is enlisted in the International Prospective Register of Systematic Reviews (PROSPERO) CRD42018108140.

\section{Eligibility criteria}

To be included in this upcoming review, studies must meet the following criteria:

1. Studies investigating the satisfaction of patients with health services in Nigerian hospitals or clinics;

2. Studies on adult patients, 18 years and above;

3. Studies performed with quantitative instruments (questionnaire-based studies);

4. The analysis will consider original research works and reviews (consideration of the latter is to ensure that no original work will be missed);

5. Eligible studies need to be published in English;
6. Studies assessing patient satisfaction as a primary outcome (see the "Outcome types" section);

7. Studies published between 2007 and 2018.

We will, therefore, exclude qualitative studies, studies published in other languages than English, and studies published earlier than 2007. The exclusion of studies published earlier than 2007 was due to the rapid changes of the Nigerian healthcare system over the last decades and to ensure studies were conducted after the introduction of the NHIS. We wanted to avoid study results which can be solely traced back to possible adaptation issues in the transition period shortly after the introduction of the NHIS.

\section{Information sources}

We will perform searches in the Medical Literature Analysis and Retrieval System Online (MEDLINE; via OvidSP), Excerpta Medica database (EMBASE; via OvidSP), Cumulative Index to Nursing and Allied Health Literature (CINAHL; via EBSCOhost), PsycINFO (PsycINFO; via OvidSP), and African Journals OnLine (AJOL; via AJOL) to retrieve primary studies and systematic reviews. For existing systematic reviews, we will also conduct searches in the Cochrane Database of Systematic Reviews (CDSR; via Wiley), the Database of Abstracts of Reviews of Effects (DARE; via Wiley), and the Health Technology Assessment Database (HTA; via Wiley).

\section{Search strategy}

Our search strategy follows a highly sensitive search approach. We will use a combination of relevant text words and Medical Subject Headings (MeSH) or other hierarchical medical vocabulary systems to incorporate basic elements of our research question, this includes population and context (e.g., hospitals, clinics, health service, and patients), geographic region (i.e., Nigeria), outcomes (e.g., patient satisfaction and perception), and study design (e.g., study, trial, and surveys). If provided by the database provider, we will use search filters to exclude animal studies and published articles before 2007. A search for MEDLINE was already developed and piloted by the author team. This MEDLINE search will be adapted to match the necessities of the other included databases. The original MEDLINE search is provided in the Appendix section below.

\section{Study selection}

Before entering the screening process, we will de-duplicate all references by using the reference software Endnote. Screening of titles and abstracts will be independently done by two of the reviewers (MA and $\mathrm{OE}$ ) in consideration of the eligibility criteria. A third reviewer's opinion (MO) will be requested to reach a general agreement in case of disagreement with regard to potential in- or exclusion of studies. The same elements of the screening process will be used for the full-text screening afterwards. 


\section{Outcome types}

The primary outcome will be patient satisfaction with health services. Studies in which satisfaction is measured only as a single dimension of service provision (as in assessing the satisfaction of patients with physician interaction) will be excluded.

\section{Data extraction}

A data extraction form will be first piloted and subsequently provided to the review team. Two reviewers (MA and $\mathrm{OE}$ ) will separately extract relevant information from the included studies. Any disagreement will be handled by discussion and final advice from a third author (MO). Data to be extracted from each study will be relevant with regard to patient-reported outcome measures (PROMs), including patient sample and results of the measurement properties (MA, OE, and MO). Thus, the following items will be considered: year, authors, sample size, study design, healthcare setting (hospital or clinic), satisfaction tool employed, dimensions of the instruments, format, and psychometric properties analyzed.

\section{Risk of bias}

The COnsensus-based Standards for the selection of health status Measurement INstruments (COSMIN) risk of bias checklist will be utilized to assess the validity and reliability of eligible studies. The checklist contains design necessities and approved statistical methods on measurement properties. For each of these properties, a checklist was developed containing standard questions needed to evaluate the quality of a study on that particular measurement property (referred to as COSMIN box). There are ten COSMIN boxes of the risk of bias checklist for the different measurement properties. For example, box 1 is for development, box 2 for content validity, and box 3 for structural validity. More than one property may be evaluated and documented in a study, meaning that more than one COSMIN box needs to be completed. The individual studies will be rated as either very good, adequate, doubtful, or of inadequate quality [27]. The quality of each study will be determined by taking the lowest rating of any of the standard questions (i.e., "the worst score counts" principle) [28]. Details and full explanation of the COSMIN checklist and its development process can be found in Mokkink et al. [29]. The use of the COSMIN risk of bias checklist is justified for this particular review as the checklist was exclusively developed and validated for evaluating the methodological quality of measures used to assess PROMs such as satisfaction, perception, and quality of life in health research [29]. Boxes of the COSMIN risk of bias statement are summarized in Table 1 .
Table 1 Boxes of the COSMIN risk of bias checklist [29]

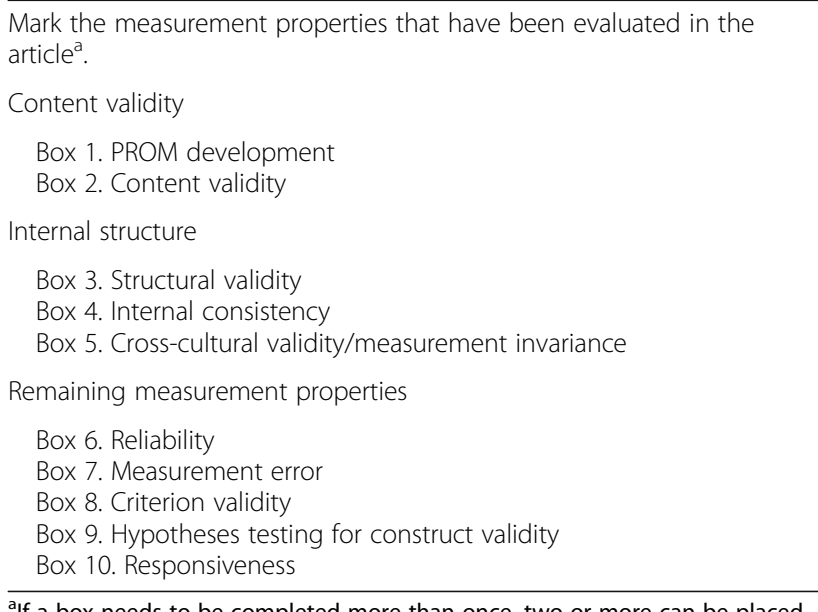

alf a box needs to be completed more than once, two or more can be placed

\section{Data synthesis}

After reviewing published systematic reviews in this research field (see the chapter "Background" section), we do not plan to do a meta-analysis as a form of data synthesis (mostly relevant to our fourth objective) as we expect studies from a wide variety of settings (e.g., general hospitals, specialized hospitals), different populations (e.g., men, women, diseased, non-diseased), different study designs (e.g., cross-sectional studies, uncontrolled before-after studies (UBAs)), and different timings of questioning the patients (e.g., hospital discharge surveys, surveys at any given time). However, a detailed narrative synthesis will be carried out to answer our four main objectives. Information will be sought only from the studies which reported results on one or more measurement properties of the COSMIN risk of bias checklist. Important features of these studies will be presented and organized in a tabular form. Included studies will be summarized under key topics that came out during thematic analysis.

\section{Discussion}

This protocol reports detailed information of an upcoming systematic review which will include studies on the satisfaction of patients with health services in Nigerian hospitals. The aim is to evaluate how patient satisfaction with health services has been described and measured in the setting of Nigerian hospitals over the past decade, a decade with fundamental healthcare reforms in Nigeria. The research gaps discovered through this systematic review will identify areas for further research as well as the direction for future studies. Areas of dissatisfaction within the context of Nigerian health services will also be pointed out. As a result, this review could contribute to a harmonization process of high-quality methods used in conducting quantitative surveys in this context. 


\section{Appendix}

Table 2 Details of one electronic bibliographic database search query (MEDLINE)

MEDLINE (via OvidSP) [1932 hits] access day 20180730

1. exp Patient centered care/

2. exp Hospitals/

3. exp Health Services Research/

4. exp Quality of Health Care/

5. clinic $^{*}$. tw.

6. hospital?.tw.

7. patient?.tw

8. outpatient?.tw.

9. inpatient?.tw

10. (medical adj1 (treatment or treatments or service or services or

centre or centres or center or centers)).tw.

11. (health adj1 (service or services or centre or centres or center or centers)).tw

12. health?care.tw.

13. health?system?.tw.

14. or/1-13

15. Nigeria.cp

16. Nigeria.tw.

17. nigerian?.tw.

18. Lagos.tw.

19. Kano.tw.

20. Ibadan.tw.

21. "Benin City".tw.

22. Jos.tw.

23. Ilorin.tw.

24. Kaduna.tw.

25. Abuja.tw.

26. Enugu.tw.

27. Warri.tw.

28. or/15-27

29. exp Patient Satisfaction/

30. acceptabilit*.tw

31. acceptance.tw.

32. attitude?.tw

33. dissatisf*.tw

34. judgement*.tw.

35. satisf*.tw.

36. view?.tw.

37. opinion*.tw

38. perception?.tw.

39. perceived.tw

40. preference?.tw.

41. preferred.tw.

42. or/29-41

43. exp Empirical Research/

44. exp Data Collection/

45. assessment?.tw.

46. evaluation?.tw.

47. intervention?.tw.

48. survey*.tw.

49. study.tw.

50. studies.tw

51. trial.tw.

52. trials.tw.

53. questionnaire?.tw.

54. or/43-53

55. 14 and 28 and 42 and 5456. limit 55 to ed="20070101-20180730"

57. (animals not (humans and animals)).sh.

58. 56 not 57

\section{Abbreviations}

AIDS: Acquired immune deficiency syndrome; CBHIS: Community-Based Health Insurance Scheme; COSMIN: COnsensus-based Standards for the selection of health status Measurement INstruments; ECHO: Economic, Clinical and Humanistic Outcomes; HIV: Human immunodeficiency virus;
LMICS: Low- and middle-income countries; NHIS: National Health Insurance Scheme; NHS: National Health Service; OOP: Out-Of-Pocket; PPPs: Publicprivate-partnerships; PRISMA: Preferred Reporting Items in Systematic Reviews and Meta-analyses; PRO: Patient-reported outcomes;

PROMS: Patient-reported outcome measures; PSPS: Patient Satisfaction with Pharmaceutical Service

\section{Acknowledgements}

Not applicable

\section{Authors' contributions}

MA and OE were responsible for designing the study, formulation of review questions, eligibility criteria, and extraction of data from included studies as well as selecting appropriate methods to synthesize data and assess the risk of bias. MO and CV contributed to the design of the study eligibility criteria. TLH selected most relevant databases and developed the final search strategy. MA wrote the first draft, and all the authors were instrumental to the manuscript corrections before the final version. All authors read and officially agreed to submit the final manuscript.

\section{Funding}

The author team received no third-party funding or external financial support for conducting this research.

\section{Competing interests}

The authors declare that they have no competing interests.

\section{Author details}

${ }^{1}$ Department of Clinical Pharmacy and Pharmacy Management, University of Nigeria, Nsukka, Enugu State, Nigeria. ${ }^{2}$ Research Group for Evidence-Based Public Health, Institute for Public Health and Nursing Research, Health Sciences, University of Bremen, Bremen, Germany. ${ }^{3}$ Leibniz Institute for Prevention Research and Epidemiology - BIPS, Bremen, Germany. ${ }^{4}$ Department of Clinical Pharmacy and Pharmacy Management, Nnamdi Azikiwe University, Awka, Anambra State, Nigeria.

Received: 1 November 2018 Accepted: 13 September 2019 Published online: 01 November 2019

\section{References}

1. Maiyaki M, Garbati M. The burden of non-communicable diseases in Nigeria; in the context of globalization. Ann Afr Med. 2014;13:1-10.

2. Jensen J. A review of public-private partnership activities in health system strengthening. In: Taylor RM, Christian J, editors. The role of public-private partnerships in health systems strengthening: workshop summary. Washington, DC: The National Academies Press; 2015. p. 67-85.

3. Meier F, Schöffski O, Schmidtke J. Public-private partnership as a solution for integrating genetic services into health care of countries with low and middle incomes. J Community Genet. 2013;4(3):309-20.

4. PharmAccess Foundation. Nigerian health sector market study report. 2014. https://www.rvo.nl/sites/default/files/Market_Study_Health_Nigeria.pdf. Accessed 18 Oct 2018.

5. PWC Nigeria. Restoring trust to Nigeria's healthcare system. 2016. https:// www.pwc.com/ng/en/assets/pdf/restoring-trust-to-nigeria-healthcaresystem.pdf. Accessed 18 Oct 2018.

6. Parmar H. Healthcare business challenges in Nigeria. 2015. https://www. linkedin.com/pulse/healthcare-business-challenges-nigeria-hemraj-parmarmba. Accessed 18 Oct 2018

7. Okogba E. Only 4\% of Nigerians are covered by NHIS. Vanguardnews 2016. https://www.vanguardngr.com/2016/12/4-nigerians-covered-nhis/. Accessed 18 Oct 2018.

8. Federal Ministry of Health. Nigerian National Health Accounts 2006-2009. http://apps.who.int/nha/database/DocumentationCentre/GetFile/51337921/ en. Accessed 18 Oct 2018

9. Krousel-Wood MA. Practical considerations in the measurement of outcomes in healthcare. Ochsner J. 1999:1(4):187-94.

10. Kozma CM, Reeder CE, Schulz R. Economic, clinical, and humanistic outcomes: a planning model for pharmacoeconomic research. Clin Ther. 1993;15(6):1121-32. 
11. Okoye M, Ukwe C, Okoye T, Adibe M, Ekwunife O. Satisfaction of HIV patients with pharmaceutical services in South Eastern Nigerian hospitals. Int J Clin Pharm. 2014;36(5):914-21.

12. Nilele A, Ukwe C, Okonta J, Ekwunife O. Development of a patient satisfaction questionnaire for HIV/AIDS patients in Nigeria. Int I Clin Pharm. 2012;34(1):98-104.

13. Karunamoorthi K, Rajalakshmi M, Makesh Babu S, Yohannes A. HIV/AIDS patient's satisfactory and their expectations with pharmacy service at specialist antiretroviral therapy (ART) units. Eur Rev Med Pharmacol Sci. 2009;13(5):331-9.

14. Ige O, Nwachukwu C. Areas of dissatisfaction with primary health care services in government owned health facilities in a semi-urban community in Nigeria. J Rural Trop Public Health. 2010;9:19-23.

15. Iloh G, Ofoedu J, Njoku P, Odu F, Ifedigbo C, Iwuamanam K. Evaluation of patients' satisfaction with quality of care provided at the National Health Insurance Scheme clinic of a tertiary hospital in South-Eastern Nigeria. Niger J Clin Pract. 2012;15(4):469-74.

16. Iloh G, Njoku P, Amadi A, Godswill-Uko E, Ofoedu J, Okafor G. Satisfaction with quality of care received by patients without National Health Insurance attending a primary care clinic in a resource-poor environment of a tertiary hospital in Eastern Nigeria in the era of scaling up the Nigerian formal sector health insurance scheme. Ann Med Health Sci Res. 2013;3(1):31-7.

17. Adekanye A, Adefemi S, Okuku A, Onawola K, Adeleke I, James J. Patients' satisfaction with the healthcare services at a north central Nigerian tertiary hospital. Niger J Med. 2013;22(3):218-24.

18. Umeano-Enemuoh J, Onwujekwe O, Uzochukwu B, Ezeoke O. Patients' satisfaction and quality of care in a tertiary institution in Southeast Nigeria. Int Res J Basic Clin Stud. 2014;2(2):14-9.

19. Iliyasu Z, Abubakar I, Abubakar S, Lawan U, Gajida A. Patients' satisfaction with services obtained from Aminu Kano Teaching Hospital, Northern Nigeria. Niger J Clin Pract. 2010;13(4):371-8.

20. Abebe T, Erku D, Gebresillassie B, Haile K, Mekuria A. Expectation and satisfaction of HIV/AIDS patients towards pharmaceutical care provided at Gondar University Referral Hospital, Northwestern Ethiopia: a cross-sectional study. Patient Prefer Adherence. 2016;10:2073-82.

21. Weston R, Dabis R, Ross JDC. Measuring patient satisfaction in sexually transmitted infection clinics: a systematic review. Sex Transm Infect. 2009; 85(6):459-67.

22. Bouzid M, Cumming O, Hunter P. What is the impact of water sanitation and hygiene in healthcare facilities on care seeking behaviour and patient satisfaction? A systematic review of the evidence from low-income and middle-income countries. BMJ Glob Health. 2018;3(3):e000648.

23. Kruk $\mathrm{M}$, Porignon $\mathrm{D}$, Rockers $\mathrm{P}$, Van Lerberghe $\mathrm{W}$. The contribution of primary care to health and health systems in low- and middle-income countries: a critical review of major primary care initiatives. Soc Sci Med. 2010;70(6):904-11.

24. Almeida R, Bourliataux-Lajoine S, Martins M. Satisfaction measurement instruments for healthcare service users: a systematic review. Cad Saude Publica. 2015;31(1):11-25

25. Ogaji D, Giles S, Daker-White G, Bower P. Systematic review of patients' views on the quality of primary health care in sub-Saharan Africa. SAGE Open Med. 2015;3:2050312115608338.

26. Shamseer L, Moher D, Clarke M, Ghersi D, Liberati A, Petticrew M, Shekelle $P$, Stewart L. Preferred reporting items for systematic review and meta-analysis protocols (PRISMA-P) 2015: elaboration and explanation. BMJ. 2015;350: g7647.

27. Mokkink $L$, de Vet $H$, Prinsen $C$, Patrick D, Alonso J, Bouter L, Terwee C. COSMIN risk of bias checklist for systematic reviews of patient-reported outcome measures. Qual Life Res. 2018;27(5):1171-9.

28. Terwee C, Mokkink L, Knol D, Ostelo R, Bouter L, De Vet H. Rating the methodological quality in systematic reviews of studies on measurement properties: a scoring system for the COSMIN checklist. Qual Life Res. 2012; 21(4):651-7.

29. Mokkink L, Prinsen C, Patrick D, Alonso J, Bouter L, De Vet $\mathrm{H}$, et al. COSMIN methodology for systematic reviews of patient-reported outcome measures (PROMs) - User Manual. 2018; https://www.cosmin.nl/wp-content/uploads/ COSMIN-syst-review-for-PROMs-manual_version-1_feb-2018.pdf. Accessed 18 Oct 2018.

\section{Publisher's Note}

Springer Nature remains neutral with regard to jurisdictional claims in published maps and institutional affiliations.

\section{Ready to submit your research? Choose BMC and benefit from}

- fast, convenient online submission

- thorough peer review by experienced researchers in your field

- rapid publication on acceptance

- support for research data, including large and complex data types

- gold Open Access which fosters wider collaboration and increased citations

- maximum visibility for your research: over $100 \mathrm{M}$ website views per year

At $\mathrm{BMC}$, research is always in progress.

Learn more biomedcentral.com/submissions 